\title{
Osteopetrosis - How much do we know?
}

\author{
Alka Chandra ${ }^{1, *}$, Naresh Chandra ${ }^{2}$, Nikhil Bhasin ${ }^{3}$, Parul Bansal Sareen $^{4}$ \\ ${ }^{1}$ Senior Specialist, ${ }^{3}$ DNB Student, ${ }^{4}$ Assistant Professor, Dept. of Anaesthesia and Critical Care, Hindurao Hospital and NDMC \\ Medical College, New Delhi, ${ }^{2} \mathrm{HOD}$, Dept. of Orthopaedics, Guru Gobind Singh Government Hospital, New Delhi, India
}

*Corresponding Author:

Email: dralkadelhi@yahoo.co.in

Received: $5^{\text {th }}$ March, 2018

Accepted: $28^{\text {th }}$ April, 2018

\begin{abstract}
Osteopetrosis (Marble Bone Disease) refers to a group of disorders of skeleton characterized by increased bone density on radiographs. We should be aware of this entity as due to difficult airway and sclerotic spine anesthetizing these patients may be a challenging task. Proper anesthesia work up and adequate preparedness can lead to successful management thus preventing the morbidity. We present here a case of osteopetrosis in which the diagnosis was missed in the pre-operative period.
\end{abstract}

Keywords: Anesthesia, Osteopetrosis, Musculoskeletal system.

\section{Introduction}

The overall incidence of osteopetrosis is difficult to estimate but autosomal recessive osteopetrosis has an incidence of 1 in 2,50,000 births and autosomal dominant osteopetrosis has incidence of 1 in 20,000 births. Diagnosis is largely based on clinical and radiographic evaluation, confirmed by gene testing wherever applicable. ${ }^{1}$ Patients with osteopetrosis suffer from difficult ventilation and intubation due to bone defects in addition to severe hemtological and metabolic impairments. Thus the management of these patients include a thorough evaluation of the affected system prior to surgery and anesthesia application must be planned accordingly. ${ }^{2}$

\section{Case Report}

We report a 26 year old female who was taken up as semi-emergency for fixation of subtrochantric fracture with history of fall. The patient weighed $40 \mathrm{~kg}$ and was $145 \mathrm{~cm}$ tall. Pre-operatively the patient gave significant history of easy fatiguability, history of meningitis at 3 years of age which was followed by decreased vision and fracture of tibia at the age of 12 years. Airway assessment revealed a high arched palate, irregular dentition, loose right premolars and Mallampati grade III. The laboratory findings were as follows: hemoglobin-9.2gm\%, white blood cells $8000 /$ cumm, platelet count of $1,40,000 /$ cumm.- Rest of the investigations showed no deviation from the normal values. A plan for spinal anesthesia for open reduction and internal fixation of fractured bone was made keeping in mind the anticipated difficult intubation and the anticipated operative time of $2 \mathrm{hrs}$. The spinal anesthesia was attempted in L3-4 space via the midline approach but inspite of repeated attempts in L3-4 and L2-3 space the subarachnoid block could not be achieved and then paramedian approach was tried which was successful and $0.5 \%$ Bupivacaine heavy $(15 \mathrm{mg})$ was deposited intrathecally. The diagnosis of osteopetrosis due to sclerotic bones and narrow medullary cavity which was missed by the junior surgeon in the emergency room was made in the operation theatre by a senior surgeon (Fig. 1 and 2). The surgery took 3 hours and blood loss was around $800-900 \mathrm{ml}$ for which blood transfusion was given.

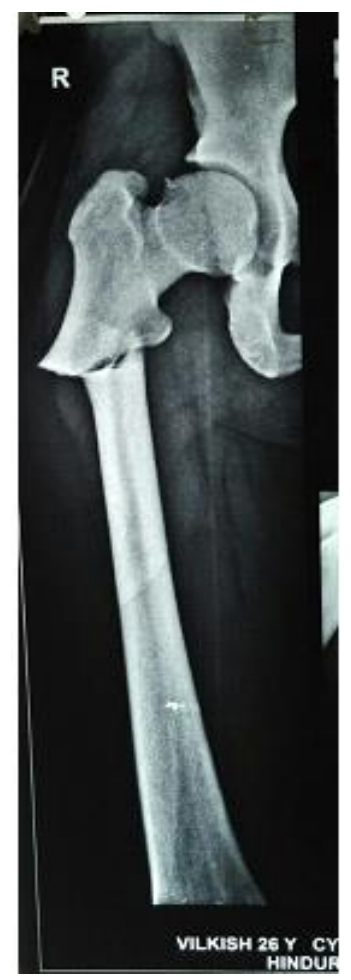

Fig. 1: X-ray showing the fracture and narrow medullary cavity 


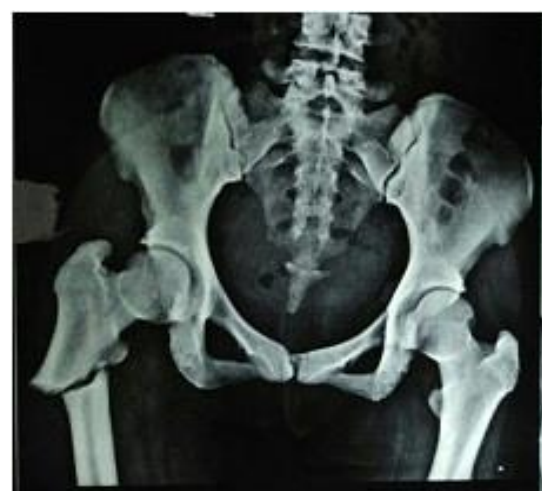

Fig. 2: X-ray pelvis with both hips showing generalized sclerosis

\section{Discussion}

Osteopetrosis vary greatly in presentation and severity ranging from neonatal onset with life threatening complications such as bone marrow failure (eg. Classic malignant Autosomal recessive Osteopetrosis) to the incidental finding of osteopetrosis on radiographs (eg.Osteopoikilosis). Classic autosomal recessive osteopetrosisis characterized by fractures, short stature, compressive neuropathies, hypocalcemia with attendant tetanic seizures and life threatening pancytopenia. The presence of primary neurodegeneration, mental retardation, skin and immune system involvement or renal tubular acidosis may point to rarer osteopetrosis variants, whereas onset of primarily skeletal manifestations such as fractures and osteomyeilitis in late childhood or adolescence is typical of autosomal dominant osteopetrosis. ${ }^{1}$

Osteoclast dysfunction in addition to normal osteoblast function results in a disproportionate increase in mineralized bone and abnormal hematopoiesis. Consequently bones are homogenously dense, sclerotic and radiopaque. ${ }^{3}$ In our case the diagnosis of osteopetrosis which was missed by the junior surgeon in the emergency room was made in the operation theatre by a senior surgeon. The anticipated difficulty in drilling due to sclerotic bone was informed to the anesthetist but the spinal anesthesia was already given by that time. The gadgets for difficult intubation were already kept ready in view of MP III, hence the anesthesiologist was mentally prepared to give general anesthesia in case the surgical time exceeded but fortunately the need to give general anesthesia did not arise.

Autosomal Dominant Osteopetrosis (ADO) which is caused by mutation in Chloride Channel 7 (CLCN 7) gene is a frequently symptomatic disease manifested by high rate of fracture, osteomyelitis, visual loss and occasional bone marrow failure. The fracture is the most prevalent consequence of $\mathrm{ADO}$, although other more severe manifestations of disease can occur and should not be confused with recessive forms of osteopetrosis particularly when identified in early childhood. ${ }^{4}$ ADO is divided into two types based on radiological findings, fracture risk and osteoclast activity. These differences suggest less brittle bone in patients with ADO I compared to that of patients with ADO II. ${ }^{5}$ Our patient had history of fracture twice, easy fatiguability due to chronic anemia. There was a history of meningitis followed by decreased vision. On examination she had a high arched palate, irregular dentition but since the diagnosis was not made by the surgeon and the x-rays of the fracture were not seen by the anesthesiologist, the diagnosis went unnoticed. Endotracheal intubation may be difficult due to abnormalities of the head and neck, including high and narrow palate, mandibular widening and hypoplasia, temperomandibular joint restriction and fractures of the cervical vertebrae., ${ }^{2,6}$

Due to difficult airway and spine the anesthetic options become limited and technically difficult in case of osteopetrosis. The bones which are sclerotic are difficult to be drilled which may escalate the drilling time. ${ }^{7}$ Repeated attempts for subarachnoid block could achieve the success in our case but had the block failed the intubation could have been equally difficult.

\section{Conclusion}

Hence, as an anesthesiologist we should be aware of this entity and its anesthetic complexities. The plans of anesthetizing the patient should be made beforehand to avoid any untoward event in the intraoperative and post-operative period. The longer surgical time and increased blood loss requiring blood transfusion must be kept in mind in these patients.

\section{References}

1. Starkz, Savarirayan R. Osteopetrosis. Orphanet J Rare Dis. 2009;4:5. doi 10.1186/1750-1172-4-5.

2. Ozer AB, Erhan OL, Demirel I, Ozcan S. Administration of general anesthesia to paediatric patient with osteopetrosis. BMJ Case Rep. 2012. doi 1136/bcr-2012006901.

3. Fasth A, Porras O. Human malignant osteopetrosis: pathophysiology, management and the role of bone marrow transplantation. Pediatr Transplant. 1993;3:102107.

4. Waque Spack SG, Hui SL, Dimeglio A, Econs MJ. Autosomal dominant osteopetrosis: clinical severity and natural history of 94 subjects with a chloride channel 7 gene mutation. J Clin Endocrinol Metab. 2007;92:771-8.

5. Von Hove RP, de Jong T, Nolte PA. Autosomal dominant type I osteopetrosis is related with iatrogenic fractures in arthroplasty. Clin Orthop Surg. 2014;6:484-8.

6. Basaranoglu G, Erden V. Difficult tracheal intubation of a patient with cervical fracture due to osteopetrosis. Paediatr Anaesth. 2001;11:745-6.

7. Sharma S, Reddy GP, Reddy WS, Ramchandran G. Marble bone disease and the Anaesthesiologist. Indian J Anaesth. 2015;59:390-1.

How to cite this article: Chandra A. Chandra $\mathrm{N}$, Bhasin N, Sareen PB. Osteopetrosis - How much do we know? Indian J Clin Anaesth. 2018;5(3):451-452. 